\title{
Group partition and restoration strategies for survivable WDM networks
}

\author{
Chen-Shie $\mathrm{Ho}^{1}$ and Sy-Yen Kuo \\ Department of Electrical Engineering \\ National Taiwan University \\ Taipei, Taiwan \\ E-mail: hocs@lion.ee.ntu.edu.tw, \\ sykuo@cc.ee.ntu.edu.tw
}

\author{
Shih-Yi Yuan \\ Department of Communications Engineering \\ Feng Chia University \\ Taichung, Taiwan \\ E-mail: syyuan@fcu.edu.tw
}

\begin{abstract}
Rather than conventional segment partition methods in link/path/sub-path based protection switching schemes, in this paper we discuss the multi-metrics resourcebased network partition approaches to meet the requirement of group switching and adapt to dynamic traffic demands. By the route selection step according to exchanged capacity estimation information, group reconfiguration process and rerouting in each group, channel utilization of network will be maximized and hence the overall quality of protection can be achieved. We consider both the static and dynamic group partition strategies under dynamic traffic demands and examine their relative influence of various network metrics. The simulation results show that resource utilization can be optimized at the expense of moderate restoration penalty under heavy load in the dynamic traffic environment over dense mesh backbone networks.
\end{abstract}

\section{INTRODUCTION}

Wavelength-routed wavelength division multiplexing (WR-WDM) network has been widely used in large-scale long-haul core networks and it is imperative that these transport networks are implemented by efficient fault tolerance mechanisms to minimize the huge avenue loss due to unpredictable failures [1][2][3]. The survivability mechanisms against different failure models in optical layer can be classified into two categories: the dynamic lightpath restoration and the preplanned protection scheme. These methods are either link/path-based or segment-based implementation [4]-[9]. All these strategies can be treated as special cases for group (submesh)-based scenarios. We summarize the relationship between these methods in Fig. 1. The path-based scheme has the benefit on resource allocation flexibility but also has the inefficiency on longer restoration cost. On the other hand, the link-based scheme has advantage in short reconfiguration time but suffers excessive resource utilization. The segment-based approach has more flexibility on routing and resource efficiency, scalability and limited restoration time. But the optimal partition to the working path of one connection may not also be optimal to other connections due to considerations for

\footnotetext{
${ }^{1}$ Chen-Shie Ho is also with Department of CSIE, Van-Nung University,
} Tao-Yuan, Chung-Li, Taiwan. E-mail: hocs@vnu.edu.tw single traffic flow, hence only local optimization can be achieved. The cycle-based protection provides the spare capacity flexibility for both link failures along and within the cycles in the network. The proposed protection group based approach in [10] don't need the pre-configuring process on spare capacity in expense of complex distributed information exchanging between network nodes by control channel and expensive but efficient resource calculation. The formation of the group is determined by current network resource status and considerations included the weight function. It can recover single link/node failure within same group and multiple failures within different groups by exploiting resources of all groups efficiently. The goal of group switching is attempting to make more efficient management for these hierarchical protection autonomous areas and completely exploits the potential resource within the protection group. Group switching can be viewed as the partition of the mesh transport network into groups to enhance the management efficiency and failure restoration. In transparent WDM network architecture with optical cross-connects (OXCs) equipped, a connection may not need to remain the same wavelength on all the links along its path, and hence more efficient wavelength resource can be achieved. In group switching restoration scenario, the traffic connection path is divided into several segments with unequal hop-length and located into several distinct group area. The path segmentation will be determined by group partition criteria. The group partition methods can be static or dynamic, which has the different influence to the quality of survivability. This paper will mainly consider the dynamic traffic condition on the wide area wavelength routed mesh topology based backbone environment with sparse wavelength conversion capability. The rest of this paper is organized as follows. Section II discusses the simulation results for the static group partition mechanism. Section III describes the proposed dynamic group partition scheme and related algorithms. The results of the simulation experiments are discussed in Section IV, Section V presents the control mechanism to adapt and optimize the signaling complexity, and the final section summarizes this paper. 


\section{STATIC GROUP PARTITION}

In static group partition scenario, the whole deployed transport network will be partitioned into several fixedsize groups. The boundary location and the dimension of the group can be determined depending on the network topology, the traffic patterns and the protection strategies. In general, from viewpoint of the network topology, $\mathrm{k}$ connect graph partition and average nodal degree-based partition can be used as the partition metrics. The kconnected subgraph will guarantee the restorability under occurrence in one of these connecting link failures, and the nodal degree examination assures the possibility of diverse routing.
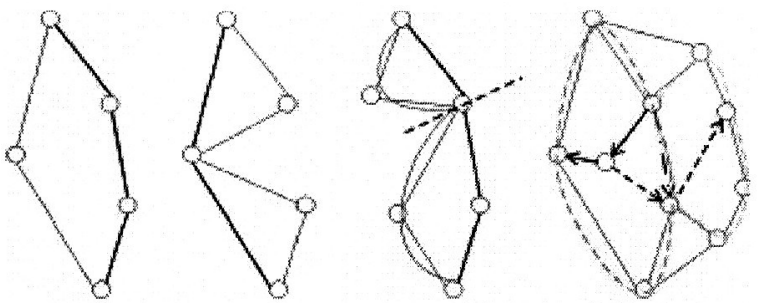

Fig. 1. Relationship between different protection schemes.

We conduct the simulation to investigate the influence of dynamic traffic demands by applying different loads to a target 34 nodes, 85 links network which has average nodal degree 3.32. The network is partitioned into 12 disjoint 2-connected groups to assure the restorability of single link failure within or on the boundary of the group The average group size is 8 nodes per group. The applied source/destination pairs are random distributed in the network. The overlapped area sharing by adjacent group is allowed for flexible spare resource allocation. The average path length for each working/protection path pair is 5.1 links. We depict the result in Fig. 2. The traffic coverage in the figure means that the degree of entire working path is covered within one group, and the fixed size $\mathrm{n}$ indicates the merged version of the grouppartitioned network. The merging of adjacent groups into a larger group indeed increases the coverage ratio but suffers the risk of unrestorability from multiple failures. From the simulation we note the following: (1) Almost $75 \%$ of all the traffic patterns are cross-grouped. This means most of the connection paths will be divided into several regions with each have pre-configured capacity allocated, and makes the survivability more complex. (2) The merging of multiple regions improves the flexibility of survivability, but the control and management architecture become more critical to achieve the message exchange and post-configuration. (3) The virtual topology formed by distinct connection requests maps into multiregion physical optical layer. Efficient mapping and grouping will produce different quality of protection. In summary, the static group partition scenario has the advantage in capacity and cost optimization, and is pertinent to static traffic demands. On the other hand, it is desirable to develop dynamic group generation which is based on the current network resource status. For example, the group size and partition with sharability will change under different traffic patterns and quality of protection demands. Also, there maybe has the possibility to further improve the performance if we substitute the nodes located at the boundary of the group domain with wavelength-conversion capability. That is, the traffics which cover number of groups will has the benefit of owing the flexibility of wavelength utilization depends on the latest network status in each group. In our method, not like the SLSP proposed in [7], the protection domain size is determined by considering the whole working path and not by segment to reduce the management overhead among various autonomous domains. In fact, our proposed method can be modified easily to accommodate varying traffic demands and adapt to different quality of protection. Also note that the restoration time variation in different protected group will cause unavailability or unacceptable failure recovery duration delay by this way. The pre-configured protection cycle (p-cycle) scheme proposed in [9] owns the mesh-like routing flexibility and ring-like restoration efficiency. We do not adopt this sort of scheme due to its high computational complexity in cycle preselection phase and resource unavailability under multiple failures.

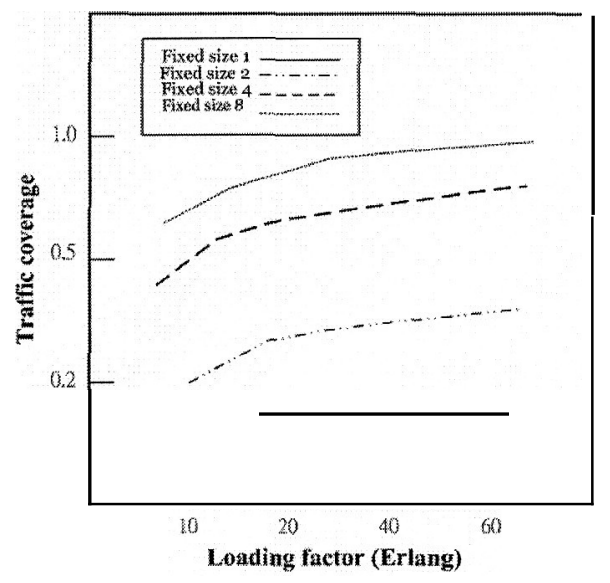

Fig. 2. Simulation result of static group partition.

\section{PROPOSED PARTITION METHOD}

In this section, we will present the resource-based network partition scheme which is suitable for dynamic traffic patterns (note that the capacity optimization in static partition method is also appropriate to incremental 
traffic pattern while just need to pay more penalty on connection transition and switching time.) The goal of the dynamic partition is to meet high restorability, high resource utilization and a simple and efficient distributed connection management method with link state protocol that is used to distribute topology information about the network should be provided. The flowchart plotted in Fig. 3 shows the framework of the proposed scheme. We discuss each of required phase and related considerations in our strategy as follows:

\section{A. Preplanningphase}

The network can be divided by some criteria optionally by maximum clique finding or k-connected component searching process. The pre-partition has the advantage of some extent degree of optimization in local resource allocation, easy to group management and differentiation of quality of protection. In our simulation, we adopt the wavelength converter partition in the target networks in this phase. The faces in the network graph are formed by these wavelength converter and the boundary OXC nodes in the network. For size or feasibility constraints, these faces can be combined to some fixed or variant size depending on the policy of network operator.

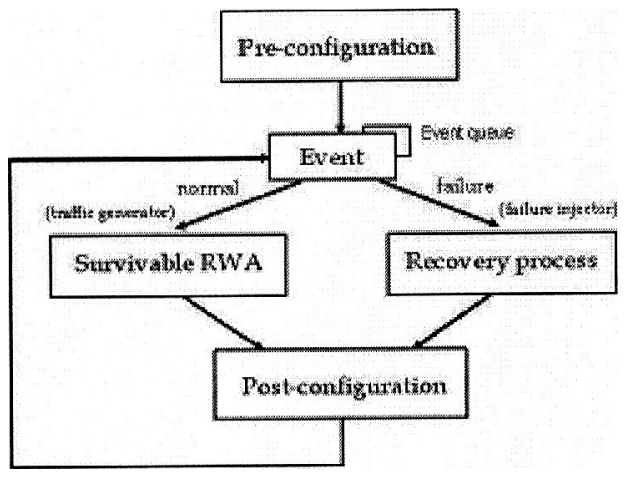

Fig. 3. Framework of survivable network system operation.

\section{B. Potential capacity calculation}

Before flooding the group resource, the maximum potential group capacity index should be examined. This value will be flooded associated with working/protection path and their wavelength information to other nodes in the same group. It is necessary in the partition process in group switching because it indicates the available resource according to the traffic distribution and resource allocation status which can be used as the routing decision for successive coming requests. The potential group capacity $C_{G}$ can be estimated as follows:

$$
C_{G}=\operatorname{Max}\left\{\sum_{i=1}^{P}\left[c_{i}-\left(\sum_{j=1} C_{P i j}+\alpha \sum_{j=1} C_{B i i}\right)\right], \sum_{i=1}^{E}\left[c_{i}-\left(\sum_{j=1} C_{P i j}\right)\right]-\alpha \sum_{j=1} C_{B i j}\right\}
$$

Here $P$ means the hop length of the longest least load path in the group, $c_{i}$ means the maximum pre-configured capacity in a link, $C_{P}$ means the capacity in link $i$ allocated to working path for connection $j, C_{B}$ means the capacity in link $i$ allocated to protection path for connection $j, E$ is the number of link in the group. Note that the protection capacity can be shared by several link or node disjoint working paths. The sharability can be determined by different QoP. The calculation metric can be either free/shared wavelength-based or hop-based. The hop length constraint may append to the capacity calculation step but it may also increase the blocking probability under heavy load condition.

\section{Post-configuration phase}

The works have to do in this phase are wavelength rerouting and cost optimization. By wavelength rerouting we can adjust the resource pre-allocated to one protection segment by local capacity optimization. Generally the wavelength (channel resource) and routes (topology resource) rerouting can be considered and performed together to further save the network resource, but we omit the route rerouting because of its higher computational complexity. In our experience, the increase in capacity saving cost about $8 \%$ but the rerouting time cost about $49 \%$, which means that rerouting cost will play an important role in time-critical restoration procedure. Wavelength rerouting to existing provisioned connections can be classified to partial rerouting and full rerouting. Contrast to the full rerouting which reassign the spare wavelength to the entire protection path, partial rerouting scenario only attempt to achieve the minimum postconfiguration cost by adjusting the wavelength used in minimal set of segments within the path. The cost optimization can be achieved by take the constraints of wavelength conversion into account.

\section{Routing and wavelength assignment}

In group switching, given the network topology, when a new connection request arrives, we first determine the working path from source to destination which can fit the wavelength continuity constraint in each group by least load path first strategy. The boundary nodes which record the capacity information will determine if it could acknowledge the connection request by examine the potential group capacity. If so, the boundary ingress node will start the route discovery and wavelength searching process. The node-disjoint protection path related to the working path will be found in each group. If the path pair can be found, the egress boundary nodes will deliver the connection request message to next ingress node in next group, send ACK message to precedent egress node, and flood the latest capacity allocation information to the neighbors. Also note that the route selection criteria here is different with [7] in that the working/protection path 
pair could not be coincided nodes. That is, the protection step can select boundary WC as egress node which has more available capacity and parallel signaling message can be issued simultaneously. Another benefit is that the risk of boundary node failure can be reduced in this way. The same process will repeat when the connection is released. The group-based routing and wavelength assignment scheme can be utilized in our method to further improve the RWA performance. The decision of source node for a connection request can be considered as three case as follows:

- Working/protection path pair can be routed on area with no group existed.

- One of working/protection path pair should be routed on area with existing groups and passing through them.

- Working/protection path pair must be routed on area with existing groups and passing through them together or separately.

The routes and wavelength assignment steps can be executed jointly. That is, the nodes in the network which are not located within at least one group will be received the information about the group status, including the group member, available wavelength, potential capacity and routes availability. The source node chooses one of the working/protection pair by best-first criteria. working path is determined, the protection path which will passing through the group in the network will be assigned to the common-shared protection ring then the automatic protection switching will be formed. As mentioned before, the adaptive protection ring expansion procedure is always standby to examine if the spare capacity will be sufficient for existed and future traffic demands. The shared index which indicates the protection congestion is recorded in a group and the record sets will be delivered to other nodes in the network for reference to choose the reliable route.

\section{E. Controlmechanisms}

The partition process can be activated either using centralized control by network manager, using boundary $\mathrm{WC}$ as resource manager, or by fully distributed control by each node in the network. For space limitation and practical implementing considerations, here we only discuss the last mechanisms. In distributed control, the resource calculation unit in each node should receive resource information from the neighbors, then the gathered information will be delivered to the RWA central unit which the configuration decision will be made there to control the passing of lightpath. The control message can be exchanged by in-band or out-of-band. As mentioned before, the dynamic resource allocation and provisioning is conducted by signaling operations riding over the control channel. The information delivered by the control channel provides the connectivity over the links in the network with the wavelength-channel utilization and thereby reflects the latest physical network topology. The amount of control signaling message exchanged and management strategies will affect the restoration time heavily. We adopt the flow-based signaling protocol to establish the message exchange mechanism. Each connection or its segment locates at specific group domain will be designated to a fixed flow identifier, and each node will store the connectivity with other nodes which are in same domain. The flow ID and its protection related resource will be recorded in the intermediate nodes through the working path when it is provisioned. There is also an additional bit to indicate if it is a normal exchange message or failure notification message. These messages can be exchanged periodically or in event-driven manner. The latest resource utilization and the routes information will be saved in the protection table. When the failure occurs, the node which detects this situation send the failure notification message to the closest node locate at the protection path, this process will then continue until the source and destination nodes are aware of the exception case and perform protection switching. By this way, the operations performed by the $\mathrm{OXC}$ nodes in the control plane includes processing for NORMAL message, UPDATE message, SETUP message and RELEASE message. We plot the message format in Fig. 4(a) and the relative control message signaling flow in Fig. $4(\mathrm{~b})$.

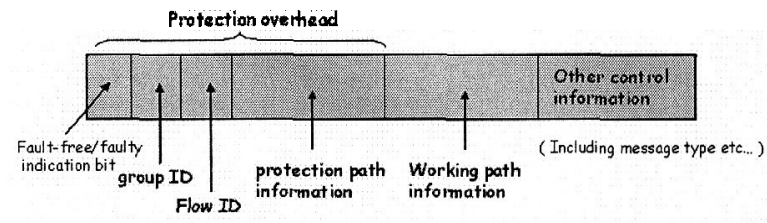

(a)

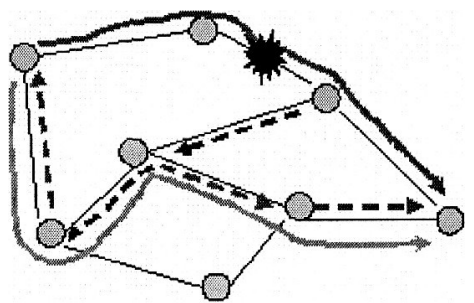

(b)

Fig. 4. Control message and restoration signaling flow in our scheme.

\section{SIMULATION STUDY}

We evaluate the effectiveness of the proposed dynamic partition algorithms by performing simulation. The characteristics of simulated networks considered here are listed in Table 1. A duplex link is comprised of two 
simplex links in the opposite directions and consists of 32 wavelength channels. Every node has various connectivity in the target network topology. The connection requests arrive at a node as a Poisson process with exponentially distributed holding time with unit mean. Every node has the same probability to be a source or a destination node for a connection request. Two types of failures, link and node failures ware injected to the network in a random manner during the simulation and then we inspect the failure recovery metrics for all affected traffics to examine the restorability. We examine 3 metrics, resource utilization, rerouting earning, and restoration penalty to measure the performance of the proposed mechanism. The resource utilization is examined limited within one protection group and expect the fully exploitation can be achieved. The rerouting earning is defined as the total saving of wavelength for successive connection requests due to adjust the channel used by current segments in the group. The restoration penalty can be evaluated either by restoration time estimation or by counting the total amount of exchanged messages. Note that the restoration time consists of transition delay which results from the event contention and processing time makes it difficult to estimate in prior. We examine restoration penalty by summing all the control messages including the failure recovery duration. The single link and node failures distributed in random manner are injected in random time period, that is, the corresponding link or node is removed from the original network and the restoration procedure is activated.

The wavelength utilization ratio versus loading factor (in Erlang unit) is plotted in Figure 6(a). The ratio can be divided into 2 types: local and global. The local utilization which means the ratio of utilizing wavelengths and fiber ports within the protection group is higher than the global ratio which means the degree of network resource accessing. We only present local utilization for the sake of the feature of the dynamic constructed group. For comparison we also plotted the result on static group partition for target network 1 in the figure. From there we can see that the wavelength resource is exhausted soon in each protection group, which will make the group reconstruction (dynamic adjustment process in group switching) activated. This ratio is not only relative to the distribution of the traffic demand but also to the degree (node connectivity) of each node. It is beneficial to dense network as the higher possibility. We also plot the result allowing group sharing, i.e., partial links or nodes in one group can be shared by multiple groups which attempting to exploit the resource more efficient. About 2\%-15\% efficiency can be achieved if the sharing size constraint is limited to $30 \%$. Figure $6(\mathrm{~b})$ shows result of the rerouting earning for the target networks. Only wavelength reroute is implemented in the rerouting phase as mentioned before. It has up to $50 \%$ efficiency in the dense network which has more available capacity resource can be reallocated. If the wavelength conversion capability is appended at node in the network, the rerouting gain will be improved apparently as showed in the figure. This brings the optimal WC allocation problem which will be studied in later research. The restoration penalty versus loading factor is plotted in Fig. 6(c). As seen from the figure, the amount of exchanged message grows quickly under the heavy load condition after the failure occurred. It consists of group operation messages exchanged in normal condition and messages required for failure recovery. This value can be reduced if the flooding and recovery signaling mechanism is made simpler and keep minimal. Also, the dense networks have significant superior as the larger successful rerouting possibility in the original or merged group. To compare the control overhead in two different control mechanism, we plotted the total control message exchanged during the whole simulation period in Fig 6(d). The unit of the control overhead is by number of exchanged message. The amount of control messages grows fast when the traffic loads increase. The centralized mechanism outperforms the distributed one in high loads situations. But as mentioned before, the restoration delay also increases in this case and the connection recovery requests from each manager affected by the failure are queued and processed in first-come-first-served manner. The partition which incorporating with the restoration latency and the manager election policy may be another choice in optimal average delay restoration requirement.
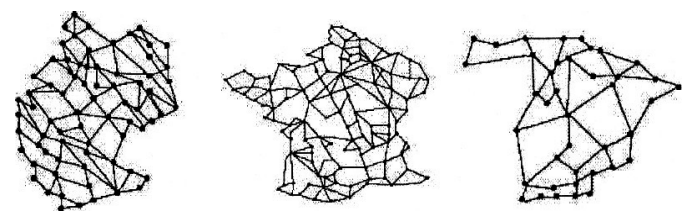

Table 1. Sample network topology information

\begin{tabular}{|c|c|c|c|}
\hline $\begin{array}{c}\text { Target } \\
\text { network }\end{array}$ & $\begin{array}{c}\text { Node } \\
\text { number }\end{array}$ & $\begin{array}{c}\text { Link } \\
\text { number }\end{array}$ & $\begin{array}{c}\text { Average group } \\
\text { size } \\
\text { (in \#node) }\end{array}$ \\
\hline (a) Germany & 73 & 130 & 12 \\
\hline (b) French & 122 & 214 & 15 \\
\hline (c) Poland & 47 & 70 & 8 \\
\hline
\end{tabular}

\section{CONCLUSION}

This paper studied the group partition based restoration heuristics in optical WDM networks. We discuss both the static and dynamic partition scenarios. The static partition mechanism has the advantage in ILP optimization and is suitable for static and incremental traffic demands. For the dynamic traffic demands, the adaptive protection 
group partition mechanism by available network capacity is more flexible and properly reflects practical condition due to the variant traffic demands. We verify the efficiency by examining various performance metrics. The results demonstrate that the proposed strategy can improve the survivability performance compared to the static partition scenario. The resource utilization can be further increased if more hardware capacity resource is implemented and well-designed control protocol can be installed on each node in the network.

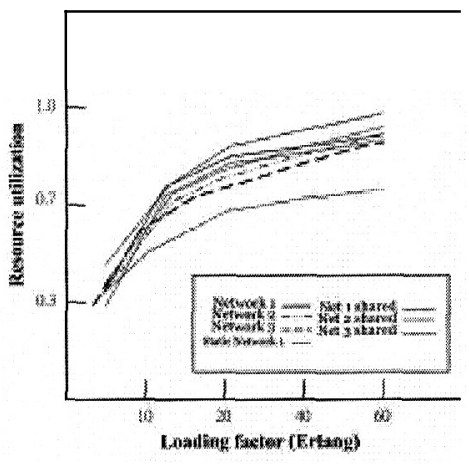

(a)

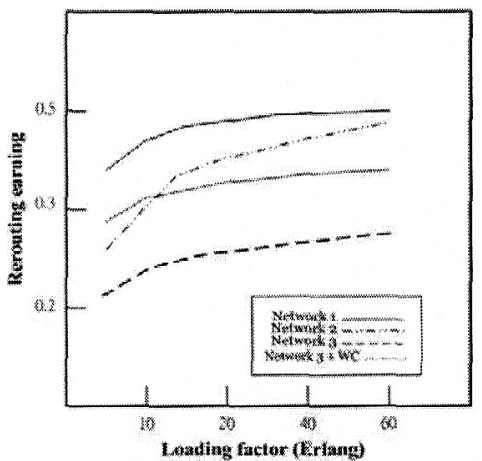

(b)

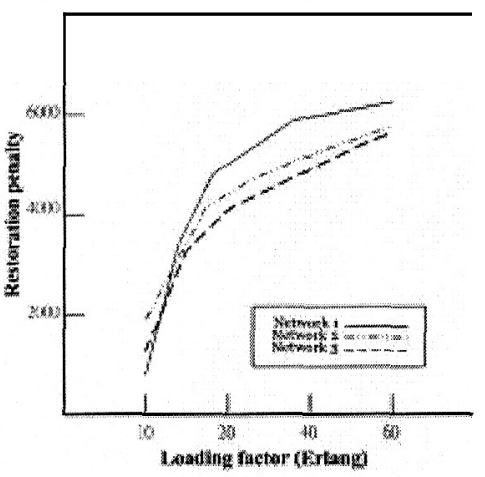

(c)

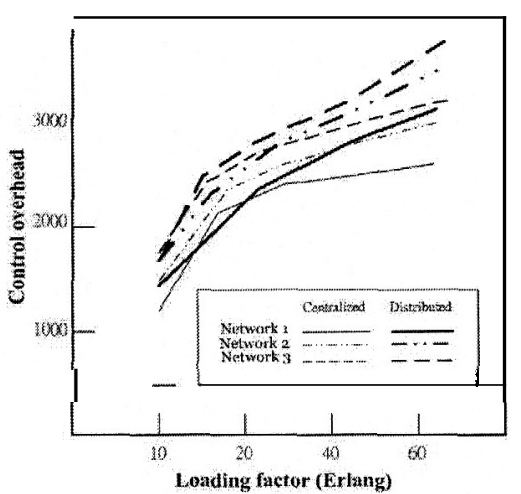

(d)

Fig. 6 . Various simulationparameters vs. loading factors.

\section{REFERENCES}

[1] H. Zang, J. P. Jue, and B. Mukherjee, "A review of routing and wavelength assignment approaches for wavelengthrouted optical WDM networks," Optical Networks Magazine, vol. 1, no. 1, January 2000, pp. 47-60.

[2] S. Ramamurthy and B. Mukherjee, "Survivable WDM mesh networks, Part 11 -Restoration", in Proc. ICC, vol. 3, 1999, pp. 2023-2030.

[3] S. Ramamurthy and B. Mukherjee, "Survivable WDM mesh networks, Part I-protection," in Proc. IEEE INFOCOM, 1999,pp. 744-751.

[4] G. Mohan, Arun K. Somani, "Routing Dependable Connections with Specified Failure Restoration Guarantees in WDM Networks", in Proc. IEEE INFOCOM, vol. 3,2000, pp. $1761-1770$

[5] V. Anand, et al., "Sub-path protection: a new framework for optical layer survivability and its quantitative evaluation", UB CSE Tech. Report, Jan. 2002.

[6] C. Qu, et al., "Sub-path protection for scalability and fast recovery in WDM mesh networks", Proc. OFC'02', Anaheim, CA, Mar. 2002, pp. 495-497.

[7] P. H. Ho and T. M. Hussein, "A framework for serviceguaranteed shared protection in WDM mesh networks", IEEE Commun. Mag., Feb. 2002, 40(2), pp. 97-103.

[8] M. Medard, S. G. Finn and R. A. Barry, "WDM loop back recovery in mesh networks", IEEE INFOCOM, 1999, PP. 744-751.

[9] W. D. Grover and D. Stamatelakis, "Cycle-oriented distributed preconfiguration: ring-like speed with mesh-like capacity for self-planning network restoration", IEEE ICC, pp. 537-543, 1998.

[10] C. S. Ho and $S$. $Y$.Kuo, "A novel restoration scheme using protection domain under dynamic traffic demands in WDM networks", ICON'03, Jeju, Korea, Feb. 2003, pp. 1597 1606. 